\title{
Take the plunge (for charity)
}

\author{
The Ice Bucket Challenge, a social media-driven fundraiser for the Amyotrophic Lateral Sclerosis (ALS) Association, \\ has raised over $\$ 100$ million to date. Despite criticisms of this fundraiser, efforts to understand why it has been so \\ successful and how to sustain its momentum would benefit its cause — research funding —and may inform future \\ charitable campaigns.
}

T he notion of dousing oneself in cold water for a charitable cause is not new, at least not to anyone who has watched people run into cold bodies of water during the winter months in so-called 'polar bear plunges' to raise funds for charity. Which might make it all the more surprising that videos of people pouring buckets of ice water over their heads, and challenging others to do the same within 24 hours and/or donate money to the ALS Association, set off a global craze this past summer. Within a month, the campaign went viral, spawning countless videos online and eliciting contributions from over 3 million donors. From July 29 to August 29, the ALS Association had raised over $\$ 100$ million in donations in response to the Ice Bucket Challenge, as compared with $\$ 2.8$ million raised during the same period in 2013.

Despite its success, the campaign has been criticized on several levels. The first criticisms focus on the intentions of the donors. It's been argued that the participants are not investing much time or effort towards the actual charitable cause and are only taking part in the challenge to achieve online notoriety, whereas other participants in the challenge may fail to donate altogether. But even if a fraction of these people were attracted by reasons of self-interest, don't the numbers speak for themselves? The aggregate outcome is undeniably positive.

Others have criticized the campaign as selling false hope of a cure and point out that it may not lead to real advances in ALS research. It may be true that the term 'cure' has been inappropriately used by many charitable organizations. But couldn't one lob the it may not lead to real advances' criticism against any research funding initiative? In the particular case of ALS, more research is exactly what is needed, as most people with this neurological disease, which is characterized by loss of motor neurons and results in paralysis, die within three to five years of diagnosis. Only one drug, riluzole, is approved by the US Food and Drug Administration for treating ALS, and it provides limited benefit regarding patient survival. Further research is needed to improve our understanding of the disease and identify new therapies to delay disease progression and extend patient survival. Genetic studies are providing insights into new genes and variants associated with the disease and potential targets for therapy. Do the variant proteins encoded by these genes alter neuronal survival in a cell-autonomous manner, or does their expression in other cell types affect neurons in a non-cell-autonomous manner? Why are particular motor neurons selectively vulnerable to degeneration while others are spared? In addition, ALS research may inform our understanding of other neurodegenerative diseases such as Alzheimer's disease, as many of the cellular and molecular mechanisms involved may be at play in multiple diseases. These indirect benefits' may be particularly helpful in advocating for funding for relatively rare disorders like ALS.

Yet another criticism of the Ice Bucket Challenge is that insufficient funds have been raised; these critics point out that research is so expensive that little progress will be made from this amount. But given the increasingly dismal funding situation and cutbacks at the US National Institutes of Health, are not any additional resources directed at research helpful? And is \$100 million (and counting) such a paltry sum, considering that in the fiscal year 2013, the National Institute of Neurological Disorders and Stroke budget was $\$ 1.53$ billion, of which only \$39 million was directed towards ALS research? Moreover, according to Charity Navigator, the ALS Association is relatively efficient, devoting $73 \%$ of its annual budget to programs and services. The ALS Association reports that last year $27 \%$ of its annual budget was devoted to research and that it funded 98 research projects. This criticism also neglects to recognize that the association devotes $51 \%$ of its funding to public policy initiatives and care services for patients with ALS. The latter funding is likely to immediately benefit those with the disease.

The final criticism is that the Ice Bucket Challenge is a one-off phenomenon rather than a sustained initiative. It's undeniable that transforming the Ice Bucket Challenge from a fad into a predictable, reliable influx of funds would enable the ALS Association to promise multiyear renewable grants, rather than one-time funding flashes, to researchers seeking to embark on expensive long-term, high-risk research projects. This transformation is, in theory, possible, and there is some precedent for this, as other disease-focused charities have demonstrated an ability to consistently raise large sums of money through annual events involving the general public. The Movember Foundation encourages men to grow their facial hair during the month of November to raise awareness for men's health issues. It was started in 2003 by 30 people and has grown steadily, with 56,129 participants raising 9.3 million Australian dollars annually in 2006 and 969,188 people raising 136.6 million Australian dollars over the course of the past year.

But what are the key ingredients that go into such a sustainable campaign? Efforts to answer that question might begin by studying and comparing the larger number of such projects initiated by other crowdfunding sites, such as Kickstarter. It's unclear whether we'll be dousing ourselves again next summer in support of ALS. But, in the meantime, focusing on understanding what made these campaigns so successful and figuring out how to maintain their momentum might be more productive than criticizing the motivations behind and the potential downstream benefits or lack thereof of any one campaign. 